\title{
High thermal neutron flux effects on structural and macroscopic properties of alkali-borosilicate glasses used as neutron guide substrate
}

\author{
R. Boffy \\ Institut Laue-Langevin, 71 avenue des Martyrs, CS 20156, F-38042 Grenoble Cedex 9, France \\ S. Peuget \\ CEA, DEN, DTCD, SECM, LMPA - Marcoule, F-30207 Bagnols sur cèze, France \\ R. Schweins, J. Beaucour \\ Institut Laue-Langevin, 71 avenue des Martyrs, CS 20156, F-38042 Grenoble Cedex 9, France \\ F.J. Bermejo \\ Instituto de Estructura de la Materia, Consejo Superior de Investigaciones Cientificas, Serrano 123, E-20886 Madrid, Spain
}

\begin{abstract}
The behaviour of four alkali-borosilicate glasses under homogeneous thermal neutron irradiation has been studied. These materials are used for the manufacturing of neutron guides which are installed in most facilities as devices to transport neutrons from intense sources such as nuclear reactors or spallation sources up to scientific instruments. Several experimental techniques such as Raman, NMR, SANS and STEM have been employed in order to understand the rather different macroscopic behaviour under irradiation of materials that belong to a same glass family. The results have shown that the remarkable glass shrinking observed for neutron doses below $0.5 \cdot 10^{18} \mathrm{n} / \mathrm{cm}^{2}$ critically depends upon the presence of domains where silicate and borate network do not mix.
\end{abstract}

Keywords: neutron guide, radiation damage, glass, neutron instruments, borosilicate, Raman, NMR, SANS, STEM

PACS: 03.75.Be, 61.80.Hg, 61.82.Ms, 81.05.Kf,

\section{Introduction}

Neutron guides are optical components widely employed in large neutron facilities running either static sources such as a nuclear reactor or at accelerator-driven centres. The surge of such now widespread devices was motivated by two technical reasons, namely to allow the installation of more scientific instruments on a single source and to lower the background noise on instrument detectors. They are used to transport thermal or cold neutrons to experimental stations that can be positioned several tens of meters away from the neutron production point. They have enabled the technique to significantly widen its scope, allowing the development of a suite of instruments able to tackle problems pertaining to a wide spectrum of disciplines such as condensed matter, neutron and nuclear physics, chemistry, materials science and engineering, bioand geosciences.

The guides are usually made of polished flat borosilicate glass coated with metallic nickel or some supermirror multilayer. The use of a boron-rich glass substrate fulfills two different purposes, first assuring the desired surface quality for the neutron reflecting material and also to absorb the neutrons that are not reflected by the mirror through $(\mathrm{n}, \alpha)$ reactions on ${ }^{10} \mathrm{~B}$.

The motivation behind our efforts comes from episodes of neutron guide failures reported from different facilities [1] [2] [3] [4]. Such failures usually result in breakup of the guide structure leading to an implosion 


\begin{tabular}{c|c|c|c|c}
\hline mol.\% & Borofloat & N-ZK7 & N-BK7 & S-BSL7 \\
\hline $\mathrm{SiO}_{2}$ & 82 & 66 & 73 & 73.5 \\
$\mathrm{~B}_{2} \mathrm{O}_{3}$ & 12 & 11.5 & 10 & 9.5 \\
\hline $\mathrm{ZnO}$ & & 10 & & \\
$\mathrm{Al}_{2} \mathrm{O}_{3}$ & 1.5 & 4 & 0.25 & \\
$\mathrm{MgO}$ & & & 1.25 & \\
\hline $\mathrm{Na}_{2} \mathrm{O}$ & 4 & 7.5 & 10 & 10 \\
$\mathrm{~K}_{2} \mathrm{O}$ & 0.5 & & 5 & 5.5 \\
$\mathrm{CaO}$ & & 1 & 0.25 & \\
$\mathrm{BaO}$ & & & 0.25 & 0.5 \\
\hline Density $\left(\mathrm{g} / \mathrm{cm}^{2}\right)$ & 2.20 & 2.47 & 2.491 & 2.50 \\
\hline
\end{tabular}

Table 1: Chemical composition and density of the studied glasses

due to loss of vacuum and the generation of a pressure wave which may transport relatively large guide debris at speeds of a few tens of meters per second, thus able to cause some significant damage. The issue is known since decades. In fact, the ILL had faced troubles with in-pile thermal-guide elements ${ }^{1}$ as early as 1978, that is less than five years after their installation. At that time, Pyrex glass had been identified as the cause of failure and was then replaced by A8866 from Corning. Later, efforts have been focused onto the understanding of the irradiation ageing of neutron supermirrors [5] [6]. Finally, the ILL restarted its investigations on this topic following guide early damages that took place on several of its beam lines (H113, $\mathrm{H} 17$ and $\mathrm{H} 25)$.

The origin of these failure episodes was promptly ascribed to radiation damage effects on the glass substrate. Within those, radiation resulting from the capture reaction ${ }^{10} \mathrm{~B}+\mathrm{n} \rightarrow{ }^{7} \mathrm{Li}+\alpha$ was assumed to be the most deleterious [7]. For some $6 \%$ of the capture reactions, the decay proceeds to the ground state of ${ }^{7} \mathrm{Li}$ whereas the remaining $94 \%$ events decay via the excited state of ${ }^{7} \mathrm{Li}$ and are accompanied by emission of a $478 \mathrm{keV}$ gamma ray. Conservation of energy and momentum gives values of $1.16 \mathrm{MeV}$ for ${ }^{7} \mathrm{Li}$ and $1.78 \mathrm{MeV}$ for the $\alpha$ particle for the ground state reaction and $0.84 \mathrm{MeV}$ for ${ }^{7} \mathrm{Li}$ and $1.47 \mathrm{MeV}$ for $\alpha$ particle generated by the gamma-ray accompanied reaction.

Both charged particles have high linear energy transfer and short range, hence deposit their kinetic energy locally within few tens of micrometers away from the point of capture. The macroscopic behaviour of this glasses under thermal neutron irradiation has been reported in a previous article [8]. Here, our aim is to describe the glass network evolution under thermal neutron flux and how it translates into changes of the macroscopic properties of the material.

\section{Materials and methods}

The four studied materials concern industry-grade alkali borosilicate glass standards, namely Borofloat, N-ZK7, N-BK7 and S-BSL7. The first three are commonly used for neutron guide manufacturing. In all cases the glass samples have been provided by different neutron guide manufacturers. As far as sample sizes are concerned, we have examined samples with typical dimensions 10x10x1.1 mm. Chemical composition and density of those glasses have been gathered in table 1.

The thermal neutron irradiations have been carried out in the T4 tube at ILL that provides an unperturbed flux of $2 \cdot 10^{13} \mathrm{n} / \mathrm{s} / \mathrm{cm}^{-2}$. Maximum sample temperature has been calculated to be about $65^{\circ} \mathrm{C}$. More details on the irradiation conditions are given in [8]. Table 2 summarizes the irradiation characteristics and damages induced by the ${ }^{10} \mathrm{~B}(\mathrm{n}, \alpha) \mathrm{Li}$ reactions. Table 3 reports the five different neutron doses that the samples were exposed to and the associated damages estimated by SRIM [9].

${ }^{1} \mathrm{H} 1$ and $\mathrm{H} 2$ systems 


\begin{tabular}{|c|c|c|c|c|c|c|}
\hline \multirow{2}{*}{$\begin{array}{c}\phi_{\text {unperturbed }} \\
\left(\mathrm{n} / \mathrm{cm}^{2} / \mathrm{s}\right)\end{array}$} & \multirow{2}{*}{$\begin{array}{l}\phi_{\text {perturbed }} \\
\left(\mathrm{n} / \mathrm{cm}^{2} / \mathrm{s}\right)\end{array}$} & \multirow{2}{*}{$\begin{array}{l}\text { Maximum temperature } \\
{ }^{\circ} \mathrm{C}\end{array}$} & \multicolumn{2}{|l|}{$\alpha$ particle } & \multicolumn{2}{|c|}{ Li ion recoil nuclei } \\
\hline & & & Displaced atoms $\left(\right.$ ion $\left.^{-1}\right)$ & range $(\mu m)$ & Displaced atoms $\left(\right.$ ion $\left.^{-1}\right)$ & range $(\mu m)$ \\
\hline $2 \cdot 10^{13}$ & $7 \cdot 10^{12}$ & 65 & 160 & $4.5-5$ & 400 & $2.4-2.6$ \\
\hline
\end{tabular}

Table 2: Informations on thermal neutron irradiation in T4 tube (ILL) and damages induced by ${ }^{10} \mathrm{~B}(\mathrm{n}, \alpha) \mathrm{Li}$. Perturbed flux measured by in-situ zirconium foil activation

\begin{tabular}{c|ccccc}
\hline Dose number & $\mathrm{F} 1$ & $\mathrm{~F} 2$ & $\mathrm{~F} 3$ & $\mathrm{~F} 4$ & $\mathrm{~F} 5$ \\
\hline Fluence $\left(\mathrm{n} / \mathrm{cm}^{2}\right)$ & $1.3 \cdot 10^{17}$ & $2.9 \cdot 10^{17}$ & $3.9 \cdot 10^{17}$ & $7.6 \cdot 10^{17}$ & $2.2 \cdot 10^{18}$ \\
$(\mathrm{n}, \alpha)$ reactions $\left(/ \mathrm{cm}^{3}\right)$ & $5.1 \cdot 10^{17}$ & $1.1 \cdot 10^{18}$ & $1.5 \cdot 10^{18}$ & $3.0 \cdot 10^{18}$ & $8.7 \cdot 10^{18}$ \\
\hline $\mathrm{E}_{\text {ioniz. }}\left(\mathrm{keV} / \mathrm{cm}^{3}\right)$ & $1.2 \cdot 10^{21}$ & $2.6 \cdot 10^{21}$ & $3.5 \cdot 10^{21}$ & $6.9 \cdot 10^{21}$ & $2.0 \cdot 10^{22}$ \\
$\mathrm{E}_{\text {nucl. }}\left(\mathrm{keV} / \mathrm{cm}^{3}\right)$ & $1.7 \cdot 10^{19}$ & $3.8 \cdot 10^{19}$ & $5.1 \cdot 10^{19}$ & $1.0 \cdot 10^{20}$ & $2.9 \cdot 10^{20}$ \\
$\mathrm{dpa}$ & 0.0041 & 0.0091 & 0.012 & 0.024 & 0.070 \\
\hline
\end{tabular}

Table 3: Thermal neutron irradiation doses and corresponding deposited energy by ionisation and nuclear interactions

A LabRAM HR800 spectrometer from Horiba with green laser (532nm) was used for the Raman analysis at CEA-Marcoule. Specifically, the irradiated samples were analysed through an optical microscope (100x objective) in a hot cell of the ATALANTE facility which was coupled to the spectrometer through an optical fiber. Small Angle Neutron Scattering experiments were carried out at the D11 instrument of the ILL suite. In that case, pristine and irradiated samples were crushed into powder and used to fill $0.5 \mathrm{~mm}$ thick cells. This was done to increase the SANS intensity as $1.1 \mathrm{~mm}$ thick samples were too absorbent. Finally, single pulse MAS-NMR experiments were done on the pristine glasses with $400 \mathrm{MHz}$ and $800 \mathrm{MHz}$ Bruker spectrometer for ${ }^{29} \mathrm{Si}$ and ${ }^{11} \mathrm{~B}$ respectively. The $800 \mathrm{MHz}$ measurements were made possible thanks to the TGIR-RMN-THC program. Details concerning the experimental parameters are reported in table 4.

\section{Results}

\subsection{Pristine glasses}

The Raman signals of the four pristine glasses are plotted in figure 1a. From the figure 1a one can see that the materials are split into two well differentiated groups. On one hand, Borofloat and N-ZK7 show Raman spectra with features characteristic of silica and borate glasses [10] [11]. Specifically, the Si-O-Si rocking and bending vibration band (named $\mathrm{R}$ band) position is very close to the one of silica glass [12]. Moreover the spectral region of Si-O stretching vibration between $\left(850\right.$ to $1200 \mathrm{~cm}^{-1}$ ) is also very similar to silica spectra with mainly two bands at 1060 and $1200 \mathrm{~cm}^{-1}$. Moreover a large band is observed in the region between 750 and $810 \mathrm{~cm}^{-1}$, with mainly two contributions at around 770 and $810 \mathrm{~cm}^{-1}$ which is characteristic of borate glasses. The first contribution is generally assigned to diborate units, a borate ring with boron in three and four fold coordination. The second contribution is generally assigned to boroxol rings, boron only in trigonal configuration [10] [13]. On the other hand, N-BK7 and S-BSL7 show Raman spectra features characteristic of borosilicate glass with a good mixing of borate and silicate units. R-band is located at higher Raman shift (around $506 \mathrm{~cm}^{-1}$ ) and the borate bands are less intense. In addition, one can notice the existence of a strong peak around $630 \mathrm{~cm}^{-1}$ that has been associated [14] [15] [16] to

\begin{tabular}{ccc}
\hline & ${ }^{11} \mathrm{~B}$ & ${ }^{29} \mathrm{Si}$ \\
\hline Static magnetic field $(\mathrm{T})$ & 18.8 & 9.4 \\
Observation frequency $(\mathrm{MHz})$ & 256.8 & 79.5 \\
Spinning frequency $(\mathrm{kHz})$ & 20 & 5 \\
Recycling time & $1 \mathrm{~s}$ & $1 \mathrm{~h}$ \\
\hline
\end{tabular}

Table 4: Experimental parameter for NMR measurements 


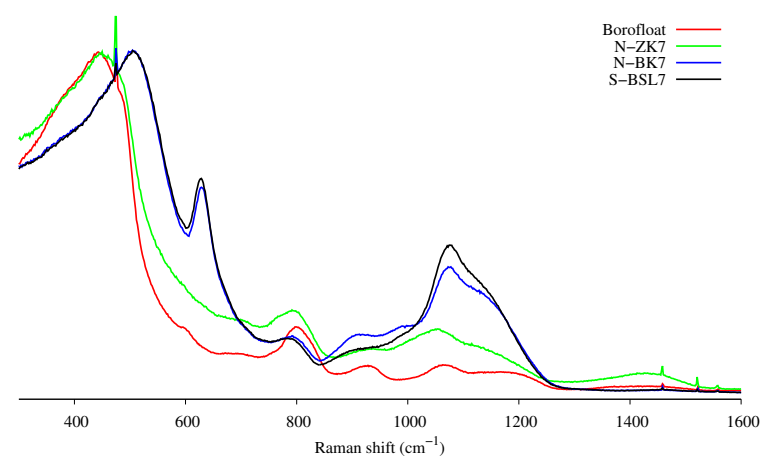

(a) Pristine glasses

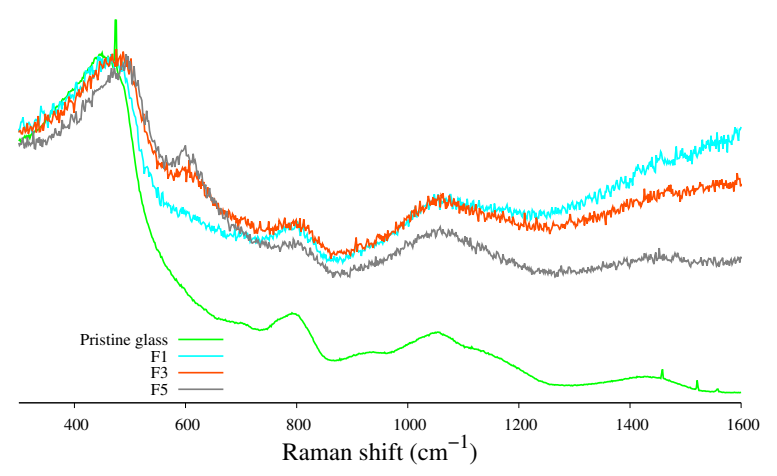

(c) N-ZK7, pristine and three doses

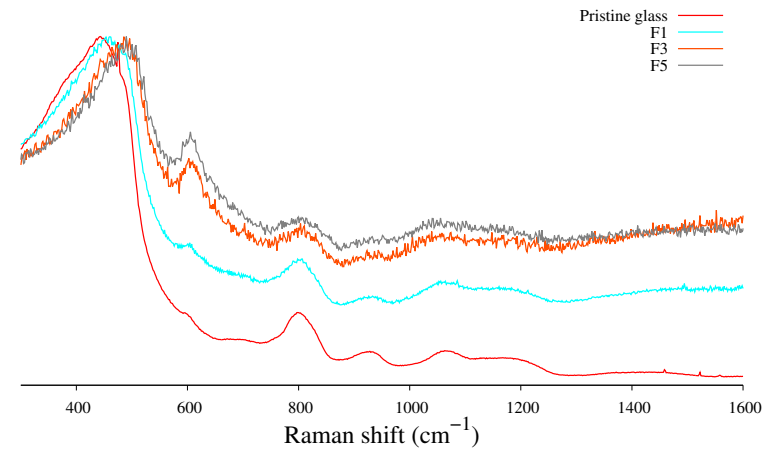

(b) Borofloat, pristine and three doses

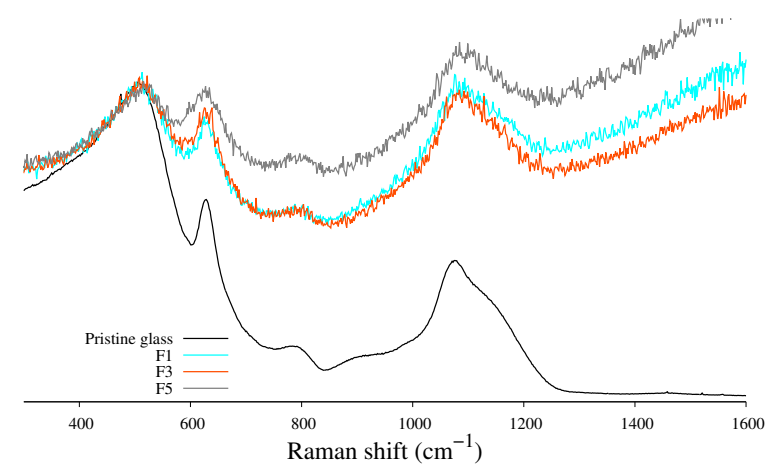

(d) S-BSL7, pristine and three doses

Figure 1: Raman spectra of the glasses, irradiated and non irradiated samples . (a) Pritine glasses, (b) Borofloat, (c) N-ZK7, (d) S-BSL7

\begin{tabular}{c|cccc}
\hline & Borofloat & N-ZK7 & N-BK7 & S-BSL7 \\
\hline N4 $(\%)$ & 23 & 30 & 91 & 90 \\
\hline
\end{tabular}

Table 5: N4 of the pristine glasses calculated from the MAS-NMR spectra with the software DMfit [21]

structures close to Reedmergnerite or Danburite crystals composed of ring of boron and silicon tetrahedra.

The ${ }^{11} \mathrm{~B}$ and ${ }^{29} \mathrm{Si}$ MAS-NMR spectra are reported in figures $2 \mathrm{a}$ and $2 \mathrm{~b}$ respectively. The measurement of ${ }^{11} \mathrm{~B}$ MAS-NMR signal with an intense magnetic field $(18.8 \mathrm{~T})$ gives a clear splitting of the $\mathrm{BO}_{3}$ triangles and $\mathrm{BO}_{4}$ tetrahedra contribution around $12 \mathrm{ppm}$ and $-2 \mathrm{ppm}$ respectively. The number of four-fold coordinated boron atoms (N4) can be directly calculated by the integration and ratio of the two peaks [17]. The value for the N4 parameter has been calculated for the different glasses and is reported in table 5. Looking at the low chemical shift, one can see in the Borofloat ${ }^{11} \mathrm{~B}$ spectra the contribution of two different sites corresponding to boron tetrahedra linked with either three or four silicon tetrahedra [16]. These two contributions are usually located around $0 \mathrm{ppm}$ and $-2 \mathrm{ppm}$ respectively. Taking this into consideration, one can notice that $\mathrm{N}-Z K 7$ has more $\mathrm{BO}_{4}(1 \mathrm{~B}, 3 \mathrm{Si})$ while $\mathrm{N}-\mathrm{BK} 7$ and $\mathrm{S}-\mathrm{BSL} 7$ have a predominance of $\mathrm{BO}_{4}(0 \mathrm{~B}, 4 \mathrm{Si})$. The ${ }^{29} \mathrm{Si}$ signal for Borofloat and N-ZK7 corresponds to mostly Q4 contributions while for the two other materials the band which is centered around -100ppm is certainly generated by a mix of Q3 and Q4 units[18] [19] [20].

Only the SANS intensities from N-ZK7 are here reported as the other materials did not show scattering patterns which can be ascribed to the presence of heterogeneities. Figure 3 shows the small angle neutron scattering signal from pristine, first and fifth irradiated dose of N-ZK7. The Q-range of the plot has been 


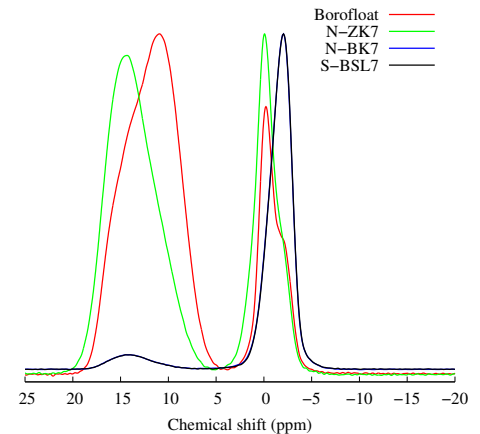

(a) MAS-NMR signal on ${ }^{11} \mathrm{~B}$ with $800 \mathrm{MHz}$ spectrometer

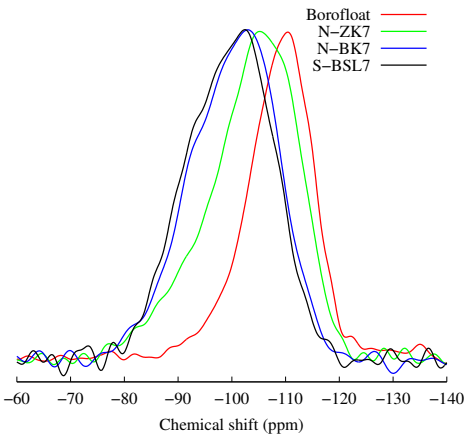

(b) MAS-NMR signal on ${ }^{29} \mathrm{Si}$ with $400 \mathrm{MHz}$ spectrometer

Figure 2: NMR spectra of irradiated and non irradiated samples

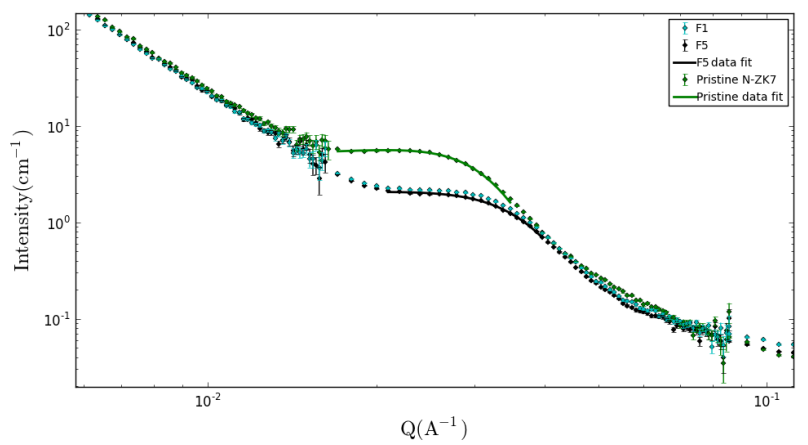

Figure 3: SANS intensity measured on D11 (ILL) on pristine, $1^{\text {st }}$ and $5^{\text {th }}$ dose of N-ZK7 glass. Ramp at low Q is caused by interfaces between powder grains and air

limited to the interesting region between 0.006 and $0.1 \AA^{-1}$ even though the real curve ranged from 0.002 to $0.5 \AA^{-1}$. The curve flattening around $0.02 \AA^{-1}$ for the pristine glass proves the existence of structures with a high enough coherent scattering density contrast to the bulk. It is possible to fit correctly [22] this zone with a hard sphere model that gives a structure radius around $10 \mathrm{~nm}$. STEM observations done on N-ZK7 have shown the existence of aggregates with higher electronic density of about the same size. In addition, STEM mapping has shown that these areas were zinc, aluminium and sodium rich, see figures 4.a-f.

\subsection{Behaviour under irradiation}

The changes in sample density as a function of the received neutron dose have been taken from Ref.[8] and are here reproduced for the sake of completeness.

The evolution of the Raman spectra as a function of the radiation dose for Borofloat, N-ZK7 and SBSL7 is reported in figures $1 \mathrm{~b}, 1 \mathrm{c}$ and $1 \mathrm{~d}$ respectively. The significant background noise over the whole bandwidth comes from the signal losses in the components (mirrors, connectors, optical fibre etc) between the microscope situated in the hot cell and the spectrometer located outside. Borofloat and N-ZK7 Raman spectra evolve in a similar way as a function of the dose. The $\mathrm{Si}-\mathrm{O}-\mathrm{Si}$ band is shifted toward higher frequencies and we can notice a growth of the D2-band around $600 \mathrm{~cm}^{-1}$ usually assigned to to three-membered silicon rings [11]. For S-BSL7, the Si-O-Si band does not show large changes and one could notice a broadening of the $630 \mathrm{~cm}^{-1}$ peak. Also, at F5, the shoulder around $600 \mathrm{~cm}^{-1}$ could be associated to an increase of three-membered rings concentration.

Measurements of the NMR spectra of irradiated samples are currently being programmed to be carried out at a suitable facility able to deal with radioactive samples. 


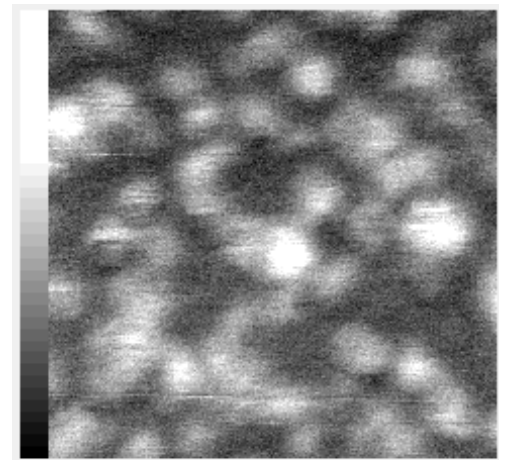

$20 \mathrm{~nm}$

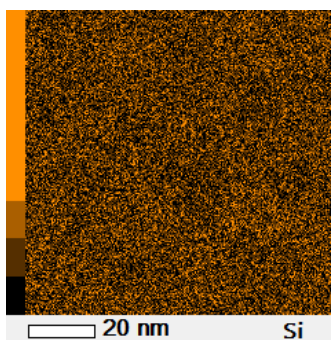

(b) Si mapping

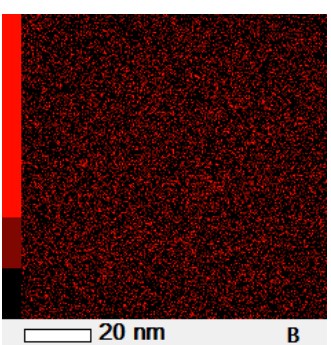

(c) B mapping

(a) STEM-HAADF image, white areas have heavier elements

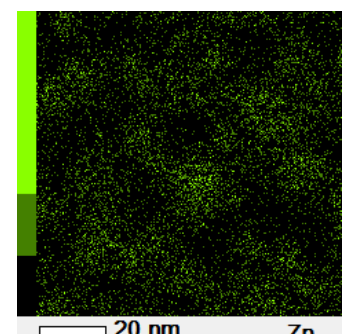

(d) Zn mapping

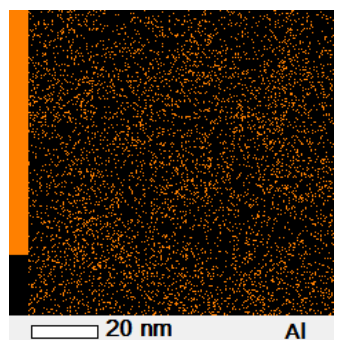

(e) Al mapping

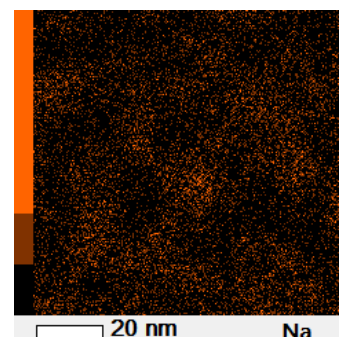

(f) Na mapping

Figure 4: STEM-HAADF imaging of N-ZK7 and element mapping of $\mathrm{Si}, \mathrm{B}, \mathrm{Zn}, \mathrm{Al}, \mathrm{Na}$

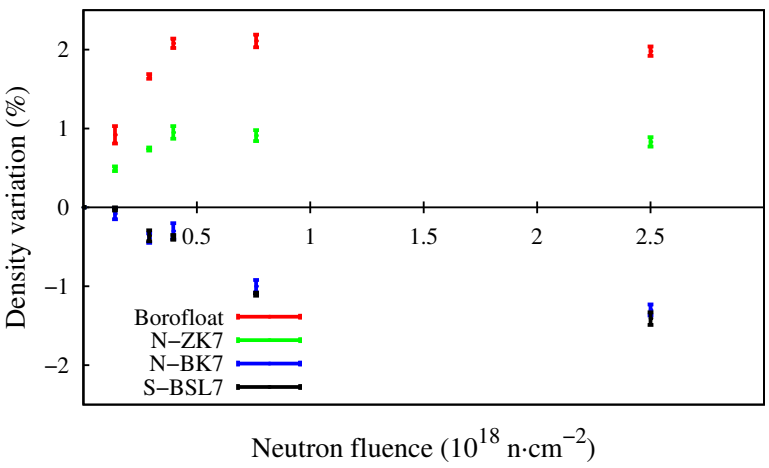

Figure 5: Density evolution of glasses as a function of received dose. Homogeneous neutron irradiation at a temperature below $65^{\circ} \mathrm{C}$. Plot extracted from $[8]$ 
Concerning the SANS intensity of the irradiated N-ZK7, whatever the dose is, the flattening of the curve is shifted toward higher $\mathrm{Q}$, around $0.025 \AA$, which indicates a reduction of the aggregate size. A similar fit yields a size of $8 \mathrm{~nm}$.

\section{Discussion}

\subsection{Pristine glasses}

The Raman and NMR spectra of the native samples provide some hints that suggest that Borofloat and N-ZK7 glasses show some clustering of silica rich and borate rich domains. Such a view is in agreement with the traditional picture of alkali-borosilicate glasses which show an alkali-borate rich phase and a silicon rich counterpart at low alkali content [23] [24] [25] [26]. From the present data we witness that the average ${ }^{29} \mathrm{Si}$ MAS-NMR chemical shift for these glasses suggests that most of silicon have four bridging oxygens and the calculation of $\mathrm{N} 4$ from ${ }^{11} \mathrm{~B}$ MAS-NMR gives a small content of NBO per remaining Si nucleus. In addition, the Raman spectra gives further support to the existence of silicate rich (Si-O-Si band near $440 \mathrm{~cm}^{-1}$ ) and borate rich areas (boroxol peak at $800 \mathrm{~cm}^{-1}$ and diborate shoulder $770 \mathrm{~cm}^{-1}$ of N-ZK7). In contrast, N-BK7 and S-BSL7 show a higher level of mixing between silica and borate units as proven by the borosilicate ring intense vibration band that shows at Raman shifts of $630 \mathrm{~cm}^{-1}$. Also, the $\mathrm{BO}_{4}$ peak of the ${ }^{11} \mathrm{~B}$ MAS-NMR spectra indicates that most of the boron atoms are surrounded by four silicons. The origin of such differences in glassy network is attributable to the network modifiers contents. Borofloat and N-ZK7 have $4.5 \%$ and $8.5 \%$ of modifiers elements respectively whereas the concentration of the other two reach $15 \%$. Alkali and alkali earth are usually used for compensating the excess of negative charge around $\mathrm{BO}_{4}^{-}$tetrahedra. But in alumino-borosilicate glasses, alkaline are preferentially used for compensating the negative charge around alumina tetrahedra. Consequently in Borofloat and N-ZK7, the amount of resulting alkali available for compensating the boron tetrahedra is very small, which explains the low value of N4 measured by NMR spectroscopy. At such low N4 value, the borate (mainly trigonal) and silicates units can not mix perfectly and create some clustering of silica rich and borate rich domains [24].

The fact that no phase segregation has been detected by SANS for Borofloat whereas Raman measurements point towards the existence of silicate and borate rich areas can be understood if the scattering contrast between both domains were too small to be detected and/or the inhomogeneities size were too small as well as some grain size effect that could mask such an intensity.

\subsection{Behaviour under irradiation}

The Raman spectra of irradiated samples develop in most cases a strong luminescent background resulting from radiation-induced colour centers that make quantitative interpretation of spectra difficult, specially for frequencies above $1000 \mathrm{~cm}^{-1}$. It is for that reason that no signal could be measured on N-BK7. It is however possible to extract useful information from features appearing at lower Raman shifts. Let's first consider S-BSL7. One sees that the spectral features are not largely affected by radiation, exception made for the luminescent background which becomes rather strong above $1300 \mathrm{~cm}^{-1}$. The $630 \mathrm{~cm}^{-1}$ peak, associated to reedmergnerite/danburite units, is almost unaffected for samples which have received the first and third doses but becomes more significant for the fifth dose. Its broadening could be associated with the growth of the D2-band which is often related to three-membered silicon rings. This effect has already been observed on a quite similar sodium borosilicate glass irradiated by the ${ }^{10} \mathrm{~B}(\mathrm{n}, \alpha)^{7} \mathrm{Li}$ reaction [27]. In parallel to this D2-band increase, a shift of the R-band of around $12 \mathrm{~cm}^{-1}$ was observed by the authors together with a swelling of around $1.5 \%$. S-BSL7 shows at F5 irradiation dose a similar swelling level and R band seems to be shifted by around $10 \mathrm{~cm}^{-1}$.

In contrast, Borofloat and N-ZK7 are much more reactive as can be inferred from changes in their density and Raman spectra. Indeed the Raman shift of the Si-O-Si peak towards higher frequencies is of around 5 times more than that of SBSL7. Moreover the density change versus dose seem to be correlated to the amplitude of the R band shift. This displacement is particularly strong for doses F1 and F3 whereas it does not show further changes between F3 and F5, a behaviour which mimics that followed by the density. 
Furthermore, the noticeable growth of the D2-band with increasing radiation dose pinpoints an important re-arrangement of the silica network. In addition the diborate and boroxol bands $\left(770 \mathrm{~cm}^{-1}\right.$ and $800 \mathrm{~cm}^{-1}$ respectively) intensity seem to decrease with increasing dose. Furukawa and White [28] showed that the R-band position was correlated to the mixing of the borate and silicate units. The important R-band shift of Borofloat under irradiation could then be a combination of silicate network shrinking and increase of mixing between silicate and borate units. Anyhow, these features support the idea that the behaviour under irradiation of Borofloat and N-ZK7 is governed in priority by their silica rich domains that represent the main phase of such clustered glasses due to the high silicon content. The higher density variation of Borofloat (2\%) compared to N-ZK7 (1\%), might be explained by its greater similarity with silica glass that shrinks up to $3 \%$ under irradiation[12]. Indeed, borofloat which have $82 \%$ of $\mathrm{SiO}_{2}$, has a R-band located at $445 \mathrm{~cm}-1$ and also well defined clustering features (D2-band and intense boroxol band).

The fact that no segregation is detected in the SANS experiment for those glasses under irradiation tells that neutron beams do not alter their homogeneity. This seems to indicate that the ballistic mixing it is not able at these fluences to induce preferential migration of elements. On the other hand, the samples which do show measurable effects at the nanoscale such as N-ZK7, the aggregate size reduction does not closely follow the density evolution, since some saturation effect is reached with the first dose. In fact, as seen from the SANS spectra the glass large-scale structure does not show further changes with increasing dose once the size of the inhomogeneities decrease from $10 \mathrm{~nm}$ to $8 \mathrm{~nm}$.

\section{Conclusion}

Characterization experiments have shown that the glasses tend to either shrink or swell under neutron flux depending on the amount of network modifiers they contain. The swelling behaviour is a slow and rather monotonous process while the shrinking is much more important and exhibits a saturation phenomenon at about $0.5 \cdot 10^{18} \mathrm{n} / \mathrm{cm}^{2}$ after which the material swells slowly. The glasses that exhibit shrinking show a clustering of silica rich and borate rich domains. The global shrinking is controlled by the behaviour of the main clustered phases i.e. the silica rich domains. This study has shown that ballistic mixing induced by the ${ }^{10} \mathrm{~B}(\mathrm{n}, \alpha){ }^{7} \mathrm{Li}$ reactions does not lead to segregation effects. For originally clustered glasses, Borofloat and N-ZK7, the Raman spectra even tend to show a better mixing of the silicate and borate units. Further analysis of the results here obtained will be done after MAS-NMR measurements on the neutron irradiated samples.

\section{Acknowledgements}

We would like to thank the guide manufacturers for the assistance they provided all along this study. Romain Boffy acknowledges financial support from the ESS-Bilbao Consortium. Financial support from the TGIR-RMN-THC Fr3050 CNRS for conducting the research is gratefully acknowledged. This work benefited from SasView software, originally developed by the DANSE project under NSF award DMR-0520547.

\section{References}

\section{References}

[1] Joint meeting of the National Organization of Test, Research, and Training Reactors and the International Group on Research Reactors, Analysis of the Causes and Consequences of Neutron Guide Tube Failures.

URL http://www.ncnr.nist.gov/trtr2005/Proceedings/

Rowe $\% 20-\% 20$ Consequences $\% 20$ of $\% 20$ Guide $\% 20$ Failure.pdf

[2] J. Beaucour, D. Bazzoli, R. Gaehler, M. Kreuz, A. Perillo-Marcone, P. Thomas, Do neutron guides live forever ?, in: $1^{\text {st }}$ Neutron Delivery System Workshop, 2006.

[3] P. Link, Neutron guides, not always perfect, FRM II news 8 (2012) 10.

[4] S. Pullen, G. Davidson, S. Pangalis, F. Klose, S. Kennedy, Report on the repair of the opal neutron beam transport system, in: Joint IGORR 2013 and IAEA Technology Meeting, 2013.

URL https://inis.iaea.org/search/searchsinglerecord.aspx?

recordsFor $=$ SingleRecord\&RN $=45103462$ 
[5] B. Ballot, Étude par réflectivité de neutrons de multicouches métalliques Nickel/Titane pour miroirs de neutrons, Ph.D. thesis, Université Paris XI (1995).

[6] K. N'Guy Maréchal, Tenue sous irradiation de supermiroirs pour guides de neutrons, Ph.D. thesis, Université Paris XI (1997).

[7] J. Paymal, M. Bonnaud, Modification des verres sous l'influence des rayonnements, in: Journées internationales d'études 1961 de l'Association belge pour favoriser l'étude des Verres et des Composés siliceux, 1961, pp. 17 - 31.

[8] R. Boffy, M. Kreuz, J. Beaucour, U. Köster, F. Bermejo, Why neutron guides may end up breaking down? some results on the macroscopic behaviour of alkali-borosilicate glass support plates under neutron irradiation, Nuclear Instruments and Methods in Physics Research B 358 (2015) 179 - 187.

[9] J. Ziegler, J. Manoyan, The stopping of ions in compounds, Nuclear Instruments and Methods in Physics Research B 35 (1988) 215-228.

[10] R. Akagi, N. Ohtori, N. Umesaki, Raman spectra of $\mathrm{K}_{2} \mathrm{O}-\mathrm{B}_{2} \mathrm{O}_{3}$ glasses and melts, J. Non-Cryst. Solids 293 (2001) $471-476$.

[11] K. Awazu, H. Kawazoe, Strained si-o-si bonds in amorphous $\mathrm{SiO}_{2}$ materials: A family member of active centers in radio, photo, and chemical responses, Journal of Applied physics 94 (10).

[12] R. Devine, Macroscopic and microscopic effects of radiation in amorphous SiO2, Nuclear Instruments and methods in Physics Research B 91 (1994) 378-390.

[13] B. Meera, A. Sood, N. Chandrabhas, J. Ramakrishna, Raman study of lead borate glasses, Journal of Non-Crystalline Solids 126 (1990) 224-230.

[14] W. Dell, P. Bray, S. Xiao, ${ }^{11} \mathrm{~B}$ NMR studies and structural modeling of na $2 \mathrm{O}-\mathrm{B}_{2} \mathrm{O}_{3}-\mathrm{SiO}_{2}$ glasses of high soda content, Journal of Non-Crystalline Solids 58 (1983) 1-16.

[15] D. Manara, A. Grandjean, D. Neuville, Advances in understanding the structure or borosilicate glasses: A raman spectroscopy study, American Mineralogist 94 (2009) 777-784.

[16] F. Angeli, T. Charpentier, D. de Ligny, B. Cailleteau, Boron speciation in soda-lime borosilicate glasses containing zirconium, Journal of American Ceramic Society 93 (9) (2010) 2693 - 2704.

[17] Y. Yun, P. Bray, Nuclear magnetic resonance studies of glass in the system Na2O-B2O3-SiO2, J. Non-Cryst. Solids 27 (1978) 363-380.

[18] J. Shneider, V. Mastelaro, E. Zanotto, B. A. Shakhmatkin, N. M. Vedishcheva, A. C. Wright, H. Panepucci, Q ${ }^{n}$ distribution in stoichiometric silicate glasses: thermodynamic calculations and ${ }^{29} \mathrm{Si}$ high resolution NMR measurements, J. Non-Cryst. Solids 325 (2003) 164-178.

[19] E. Lippmaa, M. Mägi, A. Smoson, M. T. dn G. Engelhardt, Investigation of the structure of zeolites by solide-state high-resolution ${ }^{29} \mathrm{Si}$ NMR spectroscopy, Jornal of American Chemical Society 103 (1981) 4992-4996.

[20] F. Angeli, O. Villain, S. Schuller, S. Ispas, T. Charpentier, Insight into sodium silicate glass structural organization by multinuclear NMR combined with first-principles calculations, Geochim. Cosmochim. Acta 75 (2011) 2453 - 2469.

[21] D. Massiot, F. Fayon, M. Capron, I. King, S. L. Calvé, B. Alonso, J.-O. Durand, B. Bujoli, Z. Gan, G. Hoatson, Modelling one- and two-dimensional solid-state NMR spectra, Magnetic Resonance in Chemistry 40 (2002) 70-76.

[22] SasView, http://www.sasview.org/.

[23] T. H. Elmer, M. E. Nordberg, G. B. Carrier, E. J. Korda, Phase separation in borosilicate glasses as seen by electron microscopy and scanning electron microscope, Journal of the American Ceramic Society 53 (4) (1970) $171-175$.

[24] W. B. White, Investigation of phase separation by Raman spectroscopy, J. Non-Cryst. Solids 49 (1982) 321-329.

[25] Y. Y. Galakhov, V. T. Vavilonova, Liquid-phase separation in three-component borosilicate systems, Fizika i Khimika Stekla 11 (3) (1985) 276-281.

[26] I. G. Polyakova, Alkali borosilicate systems: diagrams and properties of glasses, Physics and Chemistry of glasses 41 (5) (2000) $247-258$.

[27] S. Peuget, T. Fares, E. Maugeri, R. Caraballo, T. Charpentier, L. Martel, J. Somers, A. Janssen, T. Wiss, F. Rozenblum, M. Magnin, X. Deschanels, C. Jégou, Effect of ${ }^{10} \mathrm{~B}(\mathrm{n}, \alpha)$ Li irradiation on the structure of a sodium borosilicate glass, Nuclear Instrumentation and Methods in Physics Research B 327 (2014) 22-28.

[28] T. Furukawa, W. B. White, Raman spectroscopic investigation of sodium borosilicate glass structure, J. Mater. Sci. 16 (10) (1981) 2689-2700. 